\title{
Photosynthetic models with maximum entropy production in irreversible charge transfer steps
}

\author{
Davor Juretić*, Paško Županović \\ Faculty of Natural Sciences, Mathematics and Education, University of Split, N. Tesle 12, HR-21000 Split, Croatia
}

Received 1 July 2003; received in revised form 29 August 2003; accepted 3 September 2003

\begin{abstract}
Steady-state bacterial photosynthesis is modelled as cyclic chemical reaction and is examined with respect to overall efficiency, power transfer efficiency, and entropy production. A nonlinear flux-force relationship is assumed. The simplest two-state kinetic model bears complete analogy with the performance of an ideal (zero ohmic resistance of the P-N junction) solar cell. In both cases power transfer to external load is much higher than the $50 \%$ allowed by the impedance matching theorem for the linear flux-force relationship. When maximum entropy production is required in the transition with a load, one obtains high optimal photochemical yield of $97 \%$ and power transfer efficiency of $91 \%$. In more complex photosynthetic models, entropy production is maximized in all irreversible electron/proton (non-slip) transitions in an iterative procedure. The resulting steady-state is stable with respect to an extremely wide range of initial values for forward rate constants. Optimal proton current increases proportionally to light intensity and decreases with an increase in the proton-motive force (the backpressure effect). Optimal affinity transfer efficiency is very high and nearly perfectly constant for different light absorption rates and for different electrochemical proton gradients. Optimal overall efficiency (of solar into proton-motive power) ranges from 10\% (bacteriorhodopsin) to 19\% (chlorophyll-based bacterial photosynthesis). Optimal time constants in a photocycle span a wide range from nanoseconds to milliseconds, just as corresponding experimental constants do. We conclude that photosynthetic proton pumps operate close to the maximum entropy production mode, connecting biological to thermodynamic evolution in a coupled self-amplifying process.
\end{abstract}

(c) 2003 Elsevier Ltd. All rights reserved.

Keywords: Bacterial photosynthesis; Kinetic models; Entropy production; Power; Efficiency

\section{Introduction}

Various design principles for biological systems have been proposed, such as maximum efficiency (Stucki, 1980; Andresen et al., 2002), maximal enzymatic activity (Heinrich and Hoffman, 1991), minimum entropy production (Andriesse and Hollestelle, 2001), and other biochemical/biological objectives (Pettersson, 1996; Adami et al., 2000; Schneider, 2000). Many of the proposed optimization principles suffer from assumed separation and even opposite direction between thermodynamic and biological evolution. This leaves proposed biochemical/biological goals unconnected to physical laws directing the evolution of macroscopic systems. Life and what is good for life is considered as an end in itself not requiring any explanation outside life.

\footnotetext{
* Corresponding author. Tel.: +385-21-385-133; fax: +385-21-385-431.

E-mail address: jureti (D. Juretić).
}

We approach this problem by noticing that a high enough prescribed force can maintain a system in a far-fromequilibrium steady-state associated with a high level of entropy production. Photosynthesis is a good example. Photons have high free energy due to large temperature difference between the emitter (Sun) and the absorber (Earth). In photosynthesis, charge separation is performed, and proton electrochemical gradient is created. For instance, in the purple photosynthetic bacterium Rhodobacter sphaeroides membrane bound electron transfer proteins, the reaction center and cytochrome $\mathrm{bc}_{1}$ complex, couple electron transfer to proton release into the periplasmic space of the bacterium (Van Rotterdam et al., 2001). In even simpler photosynthesis, performed by the bacterium Halobacterium salinarium, photon free energy is directly converted into the electrochemical proton gradient by integral membrane protein bacteriorhodopsin (Lanyi and Luecke, 2001). The magnitude of a transmembrane electrochemical gradient of protons of up to $200 \mathrm{mV}$, created by the purple membrane of $H$. salinarium, is similar to the gradient created by 
photosynthetic bacteria that use bacteriochlorophyll (Van Rotterdam et al., 2002). It is only a small part of the initial free energy available in photons. All the rest has been dissipated as entropy production. From the point of view of thermodynamics high dissipation rate is always associated with photosynthesis, and it is not obvious that evolution had a goal to eliminate sources of dissipation in photosynthesis, as Lavergne and Joliot (1996) claimed. More basic question is can thermodynamics be applied at all (Hill, 1977) and if it can, are equilibrium or near-equilibrium thermodynamics appropriate tools (Meszena and Westerhoff, 1999).

Near-equilibrium irreversible thermodynamics is restricted to the region of linear relationships between forces and fluxes (Onsager, 1931a,b). The minimal entropy production theorem is valid in that region (Prigogine, 1967) and it has been often used and abused in biological applications due to unfounded expectations that biological entities would want to choose the minimal dissipation state as the next best thing to zero dissipation state (Hunt and Hunt, 1987). However, the minimal entropy production state is the steady-state with zero efficiency of free energy transduction. The dissipation in such a state is due to primary force only. Unconstrained force is maximal and it causes zero dissipation, because associated net secondary flux vanishes. Generally, unconstrained force contributes negative term to total entropy production. This effect is in fact LeChatelier-Braun principle (Kubo, 1976). It states that if a system in equilibrium is disturbed the reaction occurs in the direction which tends to decrease the cause of disturbance. When a stable steady-state with zero secondary flux is established, the destruction of input free-energy packages (photons, carbohydrates) serves only to maintain the free energy storage in the form of an electrochemical proton gradient. Such a state is appropriately labelled the static head steady-state. The static head state may have some biological relevance for resting or dormant cells and is certainly physically important as a stable nonequilibrium state close to an equilibrium state. However, in bioenergetics, one can think of the static head steady-state as the blockage to further free energy transduction due to an open circuit condition. Metabolic networks do not support such blockages.

When near-equilibrium irreversible thermodynamics is applied to bioenergetics (Stucki, 1980), it neglects the benefits that are always or often associated with nonlinear free-energy transduction in living entities. Slipping proton pumps can perform at higher overall efficiency when free-energy dissipation is increased (Juretić and Westerhoff, 1987), and always have greater energy-storage capacity for a nonlinear flux-force relationship (Juretić, 1992). Higher entropy production can actually be useful in improving regulation (Juretić and Westerhoff, 1987; Juretić, 1992). Positive feedback, or autocatalytic behaviour, has a crucial role for far-from-equilibrium nonlinear free-energy transduction in bioenergetics, as a mechanism that couples selection to augmented dissipation (Ulanowicz and Hannon, 1987).
For a continuous steady-state free-energy transduction without blockages we propose in this paper that the principle of maximum entropy production should be applied to each sequential irreversible step leading to charge separation. Maximum entropy production states are well known in physics. Kohler (1948), invoking the question posed earlier by P. and T. Ehrenfest (Enzykl. Math. Wissensch, IV, 2(II) fasc. 6 , p. 82 , note 23 ), proposed that maximal entropy production could describe steady-state processes in open systems, just as maximal entropy describes the equilibrium state of an isolated system. To describe an ideal gas not far from equilibrium, he used the Boltzmann transport equation, and assumed that extrinsic entropy production due to fixed viscosity and thermal forces must be equal to entropy production due to molecular collisions. He concluded that the steady-state distribution maximizes the intrinsic entropy production.

Ziman (1956) applied the Kohler theorem to electron transport in a solid state, also using Boltzmann's transport equation. For a fixed external electric field, he found that of all current distribution, such that the intrinsic entropy production equals the extrinsic entropy production, the steady-state distribution makes the intrinsic entropy production maximal. Ziman concluded that this theorem is mathematically equivalent to the Onsager's variational principle (Onsager, 1931a,b) and to the variational principle concerning the steady-state distribution of currents, $J_{k^{\prime}}$ through a network of resistances $R_{\mathrm{kk}^{\prime}}$, containing electromotive forces $X_{\mathrm{k}}$. This principle, formulated by Jeans more than 80 years ago (Jeans, 1923), requires that the currents be such that the function

$\sum R_{\mathrm{kk}^{\prime}} J_{\mathrm{k}} J_{\mathrm{k}^{\prime}}-2 \sum J_{\mathrm{k}} X_{\mathrm{k}}$

is a minimum, subject to the Kirchhoff's laws. Jeans imposed no condition on the rate of the heat production in resistors and the power supplied from batteries. Since these two quantities are equal in the steady-state, due to the law of energy conservation, the above function is the negative of the actual entropy production. In other words, Jeans principle states that steady-state currents of an arbitrary linear electrical network, with fixed parameters (electromotive forces and resistances), are distributed in such a way to establish the state of the maximum entropy production.

The Kohler (1948) and Ziman (1956) principles on maximum entropy production are microscopically well founded, but restricted to linear flux-force relationships. There are other thermodynamic formulations of maximum entropy production principle that apply to different far-from equilibrium physical situations with nonlinear relationships among fluxes and forces (Ziegler, 1972; Paltridge, 1979; Sawada, 1981; Swenson, 1989; Rebhan, 1990). Roderic Dewar recently provided very general information theory explanation for the selection principle of maximum entropy production (Dewar, 2003). 
Several examples of optimization for nonlinear photosynthetic models will serve to encourage the application of maximal entropy production principle in bioenergetics. We shall show that entropy production maximization, with respect to forward rate constant in each of irreversible 'productive' transitions, leads to optimal power channelling. Such steady-states are stable, have optimal performance parameters, and are biologically interesting.

\section{Materials and methods}

We describe the photosynthesis within Hill's formalism for cyclic enzymatic reactions (Hill, 1977), with Meszena and Westerhoff's thermodynamic treatment of the light absorption transition (Meszena and Westerhoff, 1999), in order to find steady-state occupational probabilities, currents, affinities, entropy production and efficiency of free-energy transduction. Hill $(1977,1989)$ assumes that reasonably stable states can be defined for the enzyme or enzymatic complex involved in a reaction cycle. The states are then associated with vertices in a diagram, while state transitions are drawn as lines connecting these states. Energy conversion can be modelled with connected loops in which free-energy transduction can occur. An essential device enabling the application of Hill's formalism to photosynthesis is the separation of light-activated transitions from radiationless and all remaining (charge-separating) dark transitions. Then the primary flux-force couple is associated with the driving light-cycle in a corresponding Hill's diagram.

We choose to vary rate constants as catalytic parameters determining transitions between neighboring states. Forward rate constants in irreversible transitions are optimized in an iterative numerical procedure. Irreversible transitions are defined as transitions with high equilibrium constant in the favour of product formation, not as transitions in which back-reactions have been neglected. The iteration consists in recalculating entropy production maximum and corresponding optimal rate constants in each such transition, by using optimal values of other rate constants in the productive pathway, until these values do not change any more. All other kinetic and equilibrium constants are kept constant. We concluded that final optimal values of rate constants are stable when small number of iterations (10 or less) produced the same final values for any initial value of forward rate constants in the wide range from $10^{-30}$ to $10^{30}$ $\mathrm{s}^{-1}$. Notice that this procedure does not produce maximal total entropy production neither in the productive pathway, nor in the whole system. However, when the system is far from the equilibrium state, the difference between overall entropy production and the sum of optimized (maximal) entropy productions in irreversible transitions, becomes, due to the nonlinear relationship between currents and affinities, of minor significance.

External forces are kept constant at predetermined values. The photon free-energy (input force) is fixed by using a monochromatic light of constant intensity. The protonmotive force (output force) is fixed by using experimental set up with constant membrane potential and constant proton gradient across photosynthetic membrane. The black lipid membrane with incorporated photosynthetic proteins is such an experimental arrangement, where both external forces are easily controlled, but good control of external forces can be also achieved in reconstituted liposomal systems (Van Rotterdam et al., 2001).

Since several different efficiency definitions are used in this work we shall list them here in order to avoid confusion. Overall efficiency $\eta$, also called free-energy transduction efficiency (Hill, 1977), or thermodynamic efficiency (Van Rotterdam et al., 2001), is the negative ratio of output to input power defined in Eq. (21). Affinity transfer efficiency is the percentage of the open circuit affinity (which is equal to the chemical potential of the photon) that may be utilized by conversion into redox energy. It is defined in this work as the $A / A_{\mathrm{oc}}$, according to symbols introduced in Eq. (3).

All kinetic models are solved in the steady-state, so that only algebraic equations are employed. All programs are written by us in FORTRAN, and are available upon request. Whenever possible, analytical calculations are performed too, in order to get better insight into numerical results. ORIGIN software is used to draw pictures. The molecular scale is used in all calculations ( $k_{\mathrm{B}}$ instead of $R$ ), but final results are expressed in the molar scale.

\section{Results}

\subsection{The two-state model for chlorophyll based photoconversion}

The very first step of photosynthetic free-energy conversion is the most important as a prerequisite for all subsequent steps. Its thermodynamic description has been recently provided (Meszena and Westerhoff, 1999). It gives the basis for non-equilibrium thermodynamics of light absorption and emission and so justifies earlier attempts to find thermodynamic limitations for photosynthesis (Knox, 1977; Juretić, 1983, 1984; Lavergne and Joliot, 1996). The common conclusion of these studies is that the chemical potential of a photon is the maximal free energy $A_{\mathrm{oc}}$ that the system (chlorophyll) at temperature $T$ can absorb when in chemical equilibrium with radiation at higher effective temperature $T_{\mathrm{R}}$ (the thermodynamic equilibrium would require $\left.T=T_{\mathrm{R}}\right)$ :

$A_{\mathrm{oc}}=h v\left(1-T / T_{\mathrm{R}}\right)$

where $h v$ is the photon energy and $T_{\mathrm{R}}$ is calculated from the assumed Planck distribution of radiation.

Assuming no volume and no entropy change between the ground $\mathrm{P}$ and excited $\mathrm{P} *$ chlorophyll state, steady-state illumination, and no branched pathway that would decrease the 
concentration of $\mathrm{P}^{*}$, the steady-state affinity $A$ of a pigment $\mathrm{P}$ would be exactly equal to (1):

$A=h v+k_{\mathrm{B}} T \ln ([\mathrm{P} *] /[\mathrm{P}])$

where $k_{\mathrm{B}}$ is the Boltzmann constant, and [P] and [P*] are fractions of ground and excited chlorophyll states respectively. A part of photon energy can be used to perform charge separation only if appropriate branched pathway exists. In the absence of such a pathway, or in the situation when net electron current $J$ through such a pathway is zero, we have an open circuit state when affinity (2) is maximal, but work (electron/proton transfer) is impossible. We know that electron acceptors and donors are located so that charge separation through a branched pathway (Fig. 1, insert) takes place with very high efficiency. With electron current $J$ flowing, dissipation occurs and steady-state affinity or photocell voltage (1) is decreased. It depends on details of a kinetic scheme and structural arrangements of a photosynthetic apparatus how much the affinity (2) will decrease from its maximal value (1). Going too far from the chemical equilibrium state defined by $A=A_{\mathrm{oc}}$ would decrease the affinity too much and extracted work would be too little. Moving too little outside state $A=A_{\mathrm{oc}}$ would produce too small a net current flow $J$ for extracting useful work. How can we determine the optimal distance from the chemical equilibrium state, i.e. the conditions for maximal free energy transfer into charge separation?

Let us use the thermodynamic force for light reactions (Meszena and Westerhoff, 1999):

$X_{\mathrm{L}}=A_{\mathrm{oc}}-\mathrm{A} \geq 0$

to define the affinity transfer efficiency as $A / A_{\mathrm{oc}}=1-X_{\mathrm{L}} / A_{\mathrm{oc}}$.

When Fig. 1. scheme is solved for the steady-state fractions $\left[\mathrm{P}^{*}\right]$ and $[\mathrm{P}]$ and Eqs. (1) and (2) used, we can find

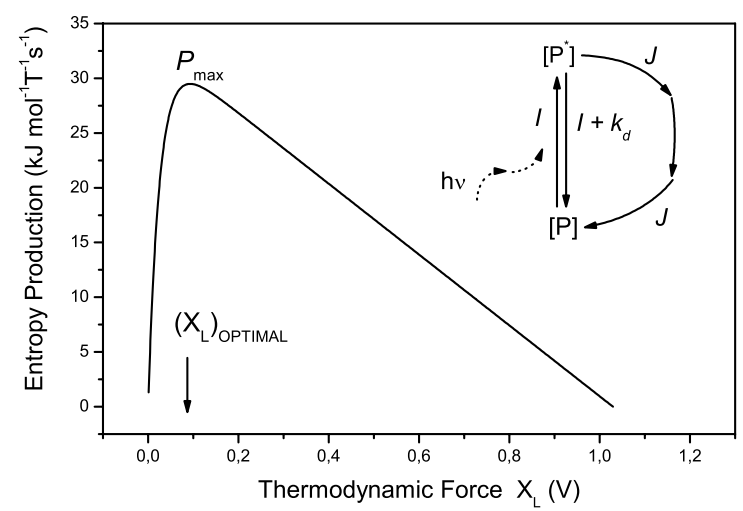

Fig. 1. Entropy production in potentially productive branch of the simple two-state model for chlorophyll-based photosynthesis. Assumed photon wavelength is $870 \mathrm{~nm}$, environment temperature $25^{\circ} \mathrm{C}$, non-radiative relaxation constant $k_{\mathrm{d}}=10^{8} \mathrm{~s}^{-1}$, and light absorption rate $I=100 \mathrm{~s}^{-1}$. Maximal entropy production state occurs for optimal thermodynamic force for light transitions and for optimal photochemical yield close to one. Insert: corresponding kinetic scheme. The excited state $\left[\mathrm{P}^{*}\right]$ relaxation can happen through the side branch where electron current $J$ flows. the dependence of $X_{\mathrm{L}}$ on photochemistry quantum yield $\Phi=J / I$, where $I$ is the flux of absorbed photons. It reads

$X_{\mathrm{L}}=-k_{\mathrm{B}} T\left\{\ln (1-\Phi)-\ln \left(1+\Phi I /\left(I+k_{\mathrm{d}}\right)\right)\right\}$

The photon flux is approximately equal to the rate constant for photon absorption, because $\left[\mathrm{P}^{*}\right] \ll[\mathrm{P}]$. The second summand in Eq. (4) is only a very small correction to the first one, so that we only need to know the photochemical yield and temperature to calculate $X_{\mathrm{L}}$. The expression (4) is also the flux $J$ dependence on thermodynamic force for light transitions when light intensity $I$ is regarded as constant. In the same approximation of neglecting small corrections $\left(k_{\mathrm{d}} \gg 1, I\right)$ :

$J=I\left\{1-\exp \left(-X_{\mathrm{L}} / k_{\mathrm{B}} T\right)\right\}$

The corresponding entropy production $P$, of the unit volume, in the productive pathway due to transmitted free energy $A$ and current $J$, is:

$T P=A J$

Taking into account Eqs. (1)-(4) we can express the dissipation (6) as a function of thermodynamic force $X_{\mathrm{L}}$ :

$T P=a(1-x)\left(1-e^{-x / b}\right) /\left(1+c e^{-x / b}\right)$

or as a function of photochemical yield $\Phi$ :

$T P=a \Phi(1+b \ln (1-\Phi)-b \ln (1+c \Phi))$

where introduced dimensionless variables are: $x=X_{\mathrm{L}} / A_{\mathrm{oc}}$, $a=I A_{\mathrm{oc}}, 1 / b=A_{\mathrm{oc}} / k_{\mathrm{B}} T, c=I /\left(I+k_{\mathrm{d}}\right)$. The dissipation is maximal for optimal $x$ (Fig. 1), and for optimal photochemical yield. Minimum entropy production would require either close to zero affinity transfer efficiency, or close to zero photochemical yield.

Approximate Eq. (5) can be written as:

$A_{\mathrm{oc}}=A-k_{\mathrm{B}} T \ln (1-\Phi)$

Assuming a small quantum yield, $\Phi \ll 1$, or an equivalently small thermodynamic force, $X_{\mathrm{L}} \ll k_{\mathrm{B}} T$, this equation can be linearized:

$A_{\mathrm{oc}} \cong A+\left(k_{\mathrm{B}} T / I\right) J$

or

$J \cong X_{\mathrm{L}} /\left(k_{\mathrm{B}} T / I\right)$

Eq. (10) offer simple interpretation in terms of the theory of electrical circuits. Then, $A_{\mathrm{oc}}$ can be identified as the electromotive force, $A$ as the voltage drop on a load, and $X_{\mathrm{L}}$ as the voltage drop through internal resistance $R_{\mathrm{i}}$. Internal and external resistances are respectively:

$R_{\mathrm{i}}=k_{\mathrm{B}} T / I$

$R=\left(\left(A_{\mathrm{oc}}-X_{\mathrm{L}}\right) / X_{\mathrm{L}}\right)\left(k_{\mathrm{B}} T / I\right)$

The entropy production (6) can be recognized now as the dissipation on the external resistor:

$T P=J\left(A_{\mathrm{oc}}-X_{\mathrm{L}}\right) \cong I\left(A_{\mathrm{oc}}-X_{\mathrm{L}}\right) X_{\mathrm{L}} / k_{\mathrm{B}} T=R J^{2}$ 
when linearized current expression (10) is used. This expression has maximal value with respect to optimal $X_{\mathrm{L}}, A$, $J$ or $R$, for the same condition:

$A_{\text {optimal }}=\left(X_{\mathrm{L}}\right)_{\text {optimal }}=A_{\text {oc }} / 2$

Maximal entropy production on the load is in accord with our proposal (see Section 2) if we consider external resistance as a variable parameter in an irreversible transition. In the steady-state maximal entropy production on the load is equal to the maximal power that can be channeled to the load. One can recognize then that Eq. (13) is equivalent to the impedance matching requirement derived from the maximum power transfer theorem (Boylestad, 1999). This requirement ensures that optimal external resistance is equal to internal resistance, so that $50 \%$ of the available source power is dissipated on the load. Optimal (and also maximal) affinity transfer efficiency $A / A_{\mathrm{oc}}$ is also $50 \%$. It is equal to power transfer efficiency due to single current passing through all elements in the loop (Fig. 1, insert).

Maximum entropy production state in the nonlinear regime (Eq. (5)) is associated with much higher optimal values for affinity transfer efficiency and for photochemical yield. Fig. 2. represents the voltage-current graph in the case of constant light intensity of monochromatic light. Using the same input values as for the Fig. 1. calculations we find from Eqs. (7) and (8) that optimal $A / A_{\mathrm{oc}}=0.91$ and optimal $\Phi=0.97$. High performance parameters are general feature of the the maximum entropy production state whenever we have strongly nonlinear and convex graph of the current versus transmited free-energy. Optimal photochemical yield is similar to the experimentally observed value for isolated reaction centers (Cho et al., 1984;

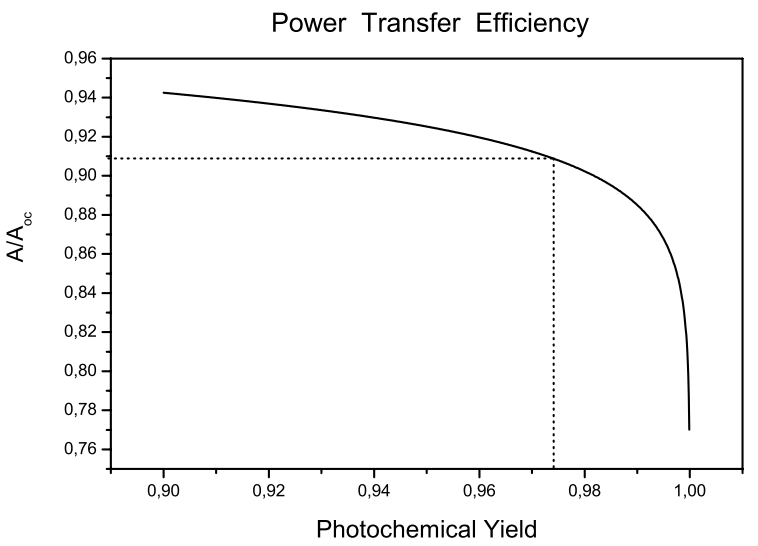

Fig. 2. Power transfer efficiency as a function of photochemical yield. The Eq. (4) is used in the two-state model for chlorophyll-based photosynthesis to find the dependence of the affinity transfer efficiency $A / A_{\mathrm{oc}}$ on photochemical yield $J / I$. Photon free-energy (open-circuit affinity) $A_{\mathrm{oc}}$ and light absorption rate $I$ are assumed to be constant. Nonlinearity allows power transfer to be more that $90 \%$ efficient (horizontal dotted line), when optimal photochemical yield is found (vertical dotted line) from the requirement for maximum entropy production in the charge separation pathway. The input values are the same as described in the Fig. 1 legend.
Lavergne and Joliot, 1996, 2000). With optimal quantum yield close to one, only about $10 \%$ of available power is dissipated in the pathway $\mathrm{P} \rightarrow \mathrm{P}^{*}$ where energy utilisation cannot occur (the vertical transition in the scheme from Fig. 1). It follows that entropy production (6) in a productive pathway is about $90 \%$ of the total entropy production. Maximal power transfered to the load is then $90 \%$ of the input power, instead of $50 \%$ as in the linear case.

With the origin of the coordinate system shifted to point $(0,0)$ in Fig. 2 it is easy to see that the area within dotted line boundary corresponds to output power, while the area just above it, until $100 \%$ efficiency is reached, corresponds to the power dissipated by the thermodynamic force for light reactions $X_{\mathrm{L}}$ on internal resistance. The introduction of $X_{\mathrm{L}}$ for photosynthesis (Meszena and Westerhoff, 1999) is very useful, because its value tells us immediately how much of the photon free energy (1) has been wasted without ever reaching the load where it can perform some useful work. In our case optimal thermodynamic force for light transitions is equal to $k_{\mathrm{B}} T_{\mathrm{R}}$, or in the molar scale $\left(X_{\mathrm{L}}\right)_{\text {optimal }}=9 \mathrm{~kJ}$ $\mathrm{mol}^{-1}$ or $0.093 \mathrm{~V}$ (Fig. 1).

\subsection{The analogy with a solar cell}

Before we discuss more complex kinetic models it would be of interest to compare the thermodynamics of the two state photosynthetic system with the interface-type photovoltaic device. The solar batery or solar cell is a $\mathrm{p}-\mathrm{n}$ junction device utilizing a photovoltaic effect (Green, 1982). Photon absortion leads to the generation of hole-electron pairs near the junction and to charge separation due to built-in field of the contact potential. The open circuit voltage of silicon cells in full sunlight is about $0.6 \mathrm{~V}$. This corresponds to open circuit voltage $A_{\mathrm{oc}}$ in Eq. (1) for photosynthesis. The reduction of the potential drop across the junction under peak power conditions is about $0.2 \mathrm{~V}$. It corresponds to thermodynamic force for light transitions introduced in Eq. (3). The output voltage of silicon cell, of about $0.4 \mathrm{~V}$, corresponds to affinity (2).

Analogy becomes quantitative when the flux-force relationship of the thermodynamical model and the currentvoltage relationship of a solar cell are compared. The net current in a solar cell is:

$J=J_{\mathrm{s}}-J_{0}\left(\exp \left(e U / k_{\mathrm{B}} T\right)-1\right)$

where the second contribution to $J$ is the ordinary current through the diode, which is saturated at the $J_{0}$ value, while the first contribution $J_{\mathrm{S}}$ is the reverse photocurrent due to the minority carriers generated optically within a few diffusion lengths of the junction. The voltage drop on the $\mathrm{p}-\mathrm{n}$ junction is $U$. The exponential expression is much greatear than one. When one is neglected in Eq. (14):

$e U=k_{\mathrm{B}} T\left[\left(\ln (1-\Phi)-\ln \left(J_{0} / J_{\mathrm{S}}\right)\right]\right.$

where $\Phi=J / J_{\mathrm{S}}$ 
Under open circuit conditions $\Phi=0$ and electromotive force is found as

$\varepsilon=-\left(k_{\mathrm{B}} T / e\right) \ln \left(J_{0} / J_{\mathrm{s}}\right)$

so that:

$e(\varepsilon-U)=-k_{\mathrm{B}} T \ln (1-\Phi)$

The last expression (17) is in complete formal analogy with Eq. (9), if electromotive force $\varepsilon=A_{\mathrm{oc}} / e$, if output voltage $U=A / e$, and if the potential reduction $\varepsilon-U=X_{\mathrm{L}} / e$.

In the steady-state, power dissipated on the load is equal to free-energy dissipation and (when devided by absolute temperature) to entropy produced on external resistance. For nonlinear $U-J$ relationship which follows from Eq. (14):

$U=\left(k_{\mathrm{B}} T / e\right) \ln \left(\exp \left(e \varepsilon / k_{\mathrm{B}} T\right)-J / J_{0}\right)$

power transfer efficiency can be much higher than $50 \%$. One can easily show that convex $U-I$ graphs will always give power transfer efficiency higher than 50\%.

The linearization is justified only in the case of very small voltages. In that case net current $J$ is proportional to the potential reduction, and the $U J$ product becomes maximal for $U_{\mathrm{opt}}=\varepsilon / 2$. Power transfer efficiency, which is $U / \varepsilon$ in this case, then reaches the maximal value of $50 \%$.

\subsection{The three-state model for chlorophyll-based photoconversion}

Charge separation is not really performed in the two state model, because the model does not contain the mechanism for the creation of an electric field and for the creation of a proton gradient across a cellular membrane that would be able to survive the cycle completion.

To see how the dissipation is channelled between different branched pathways, we must consider a slightly more complex scheme than the Fig. 1, scheme. The Hill's diagram method $(1977 ; 1989)$ and Kirchhoff's laws will be used to analyse the Fig. 3 scheme (see Section 2).

A detailed way of splitting up entropy production is to associate entropy production with each transition between two connected states in a diagram:

$T P=\sum A(\vartheta) J(\vartheta)$

where $J(\vartheta)$ is the current associated with the affinity $A(\vartheta)$ in the transition $\vartheta$.

Each term in Eq. (19) is positive definite and can be regarded as the entropy production associated with the transition $\vartheta$. This follows from the requirement, known as De Donder's Theorem (De Donder and Van Rysselberghe, 1936), that affinity and corresponding velocity always have the same sign.

The second possibility of splitting the entropy production is less detailed but better suited to connect theory to experiments. Operational input and output couples of forces and fluxes are introduced as $X_{\mathrm{i}} J_{\mathrm{i}}$ and $X_{\mathrm{o}} J_{\mathrm{o}}$. For only one input and output couple, free energy dissipation becomes:

$T P=X_{\mathrm{i}} J_{\mathrm{i}}+X_{\mathrm{o}} J_{\mathrm{o}}$

The output term can be negative. In bioenergetices it is usually the work performed against electrochemical proton gradient. In our context we shall denote the $X_{\mathrm{o}} J_{\mathrm{O}}$ as the power production. Notice that maximal power output is no longer coincidental with the maximal entropy production, because the power output contributes a negative term to the total entropy production. Overall efficiency or efficiency of free-energy transduction is then:

$\eta=-X_{\mathrm{o}} J_{\mathrm{o}} / X_{\mathrm{i}} J_{\mathrm{i}}$

In the case of the scheme shown in Fig. 3, there are four transitions, three cycles, and two operational fluxes and forces. The common cycle $\mathrm{c}$ is associated with both input and output forces. In terms of transition affinities and currents entropy production (19) is:

$$
\begin{aligned}
T P= & A_{\mathrm{PP} *}(\mathrm{~L}) J(\mathrm{~L})+A_{\mathrm{P} * \mathrm{P}}(\mathrm{D}) J(\mathrm{D})+A_{\mathrm{P} * \mathrm{P}} \\
& +(\mathrm{B} 1) J(\mathrm{~B} 1)+A_{\mathrm{P}^{+} \mathrm{P}}(\mathrm{B} 2) J(\mathrm{~B} 2)
\end{aligned}
$$

where L, D, B1 and B2 are transitions defined in the Fig. 3, legend. Due to Kirchhoff's current law in a steady-state, transition currents are connected:

$$
J(\mathrm{~L})=J(\mathrm{D})+J(\mathrm{~B} 1) \quad J(\mathrm{~B} 1)=J(\mathrm{~B} 2)=J(\mathrm{~B})
$$

where we have used the clockwise direction as the positive orientation.

The affinity $A_{\mathrm{PP} *}(\mathrm{~L})$ can be recognized as the thermodynamic force $X_{\mathrm{L}}$ in the light-activated transition introduced in

\section{Chlorophyll-Based 3-State Kinetic Model}

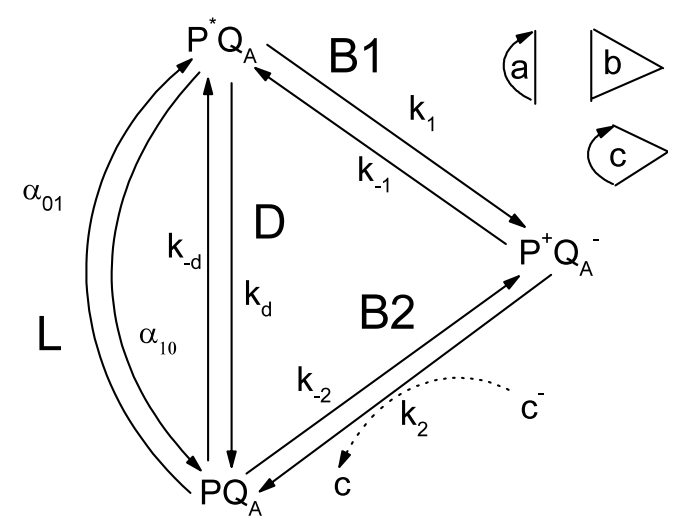

Fig. 3. The three-state kinetic model for chlorophyll-based photosynthesis. Four transitions are: light-activated transition $\mathrm{L}$ between chlorophyll ground $(\mathrm{P})$ and excited state $\left(\mathrm{P}^{*}\right)$, non-radiative transition $\mathrm{D}$ back to ground state, relaxation B1 from excited state with electron transfer and charge separation, and recovery transition B2 in which electron transfer is coupled to proton transport and to creation of proton-motive secondary force. The $\mathrm{Q}_{\mathrm{A}}$ is ubiquinone electron acceptor. The photon free energy (1) is the input force in the cycle a, while cycle b is responsible for the creation of proton electrochemical gradient (insert). 
Eq. (3). Kirchhoff's loop rule gives the connection between affinities and forces in each loop:

$$
\begin{aligned}
A_{\mathrm{P} * \mathrm{P}}(\mathrm{D})= & A_{\mathrm{oc}}-X_{\mathrm{L}} \\
& A_{\mathrm{P} * \mathrm{P}}+(\mathrm{B} 1)+A_{\mathrm{P}^{+} \mathrm{P}}(\mathrm{B} 2)-A_{\mathrm{P} * \mathrm{P}}(\mathrm{D}) \\
= & X_{\mathrm{sec}}
\end{aligned}
$$

where primary force $A_{\mathrm{oc}}$ and secondary force $X_{\mathrm{sec}}$ can be derived by forming the clockwise and counterclockwise products of rate constants in cycles $a$ and $b$ where these forces are respectively operational (Hill, 1977):

$$
\begin{aligned}
& A_{\mathrm{oc}}=k_{\mathrm{B}} T \ln \left(\alpha_{01} k_{\mathrm{d}} / \alpha_{10} k_{-\mathrm{d}}\right) \\
& X_{\mathrm{sec}}=k_{\mathrm{B}} T \ln \left(k_{1} k_{2} k_{-\mathrm{d}} / k_{-1} k_{-2} k_{\mathrm{d}}\right)
\end{aligned}
$$

It is easy to verify that photon free energy (25) is indeed given by Eq. (1). This result follows from the assumption that the energy gap between chlorophyll excited and ground state is equal to photon energy $h v$, that equilibrium constant $K(\mathrm{~L})=\alpha_{10} / \alpha_{01}$ represents the equilibrium of the system with radiation at effective temperature $T_{\mathrm{R}}$ (between 800 and $1200 \mathrm{~K}$, depending on light intensity), and that the equilibrium constant $K(\mathrm{D})=k_{\mathrm{d}} / k_{-\mathrm{d}}$ represents the equilibrium of the system with the environment heat bath at about $300 \mathrm{~K}$.

One can now use Eq. (22) to derive the entropy production expression in terms of input and output fluxes and forces:

$T P=A_{\mathrm{oc}} J(\mathrm{~L})+X_{\mathrm{sec}} J(\mathrm{~B})$

Corresponding efficiency expression (21) is of course:

$\eta=-X_{\mathrm{sec}} J(\mathrm{~B}) / A_{\mathrm{oc}} J(\mathrm{~L})$

It represents the efficiency of the input power channelling into the output power.

Since we have more detailed description now in the three state model, we can explore the dependence of a chosen transition term in Eq. (22) on a corresponding forward rate constant, taking care to maintain the same energy gap between two states connected with the chosen transition. For instance, the equilibrium constant for the B1 transition is determined as: $K(\mathrm{~B} 1)=k_{1} / k_{-1}=\exp \left(-u+h v / k_{\mathrm{B}} T\right)$, where electron donor/acceptor ratio is equal to $\exp (u)$.

With all equilibrium constants fixed, then all forces are constant too (Hill, 1977), but power transfer to any particular transition can still vary when kinetic constants in that transition are varied. We used the maximum entropy production principle in an iterative procedure to optimize power channelling in the productive pathway. Optimal $k_{2}$ is found by looking for the maximal entropy production in the B2 transition for a given $k_{1}$. Then, optimal $k_{1}$ is found by looking for the maximal entropy production in the B1 transition for a given optimal $k_{2}$. Then, optimal $k_{2}$ is found by looking for the maximal entropy production in the B2 transition for a given optimal $k_{1}$. These iterative cycles (less than 5) produced stable steady-state independent of chosen initial value for the $k_{1}$ (see Section 2). Several examples of optimization in the power transfer by using this principle are shown in the
Table 1. Notice that optimal forward rate for the recovery step is eight orders of magnitude smaller then optimal forward rate for the relaxation step, while optimal performance parameters are close to maximal possible values.

\subsection{The conditions needed for maximum to occur}

Is it always possible to find maximal entropy production associated with certain transitions in a kinetic model? Exact calculation for the three-state kinetic model (Fig. 3) gives conditions for the entropy production maximums to occur in the transitions $\mathrm{B} 1$ and $\mathrm{B} 2$, respectively, of the charge separating pathway:

$$
\begin{array}{ll}
\mathrm{B} 1 & K(\mathrm{~B} 1) K(\mathrm{~B} 2)>\left(\alpha_{10} / \alpha_{01}\right)^{2} \\
\mathrm{~B} 2 & K(\mathrm{~B} 1) K(\mathrm{~B} 2)>\left(k_{\mathrm{d}}+\alpha_{10}\right) / \alpha_{01}
\end{array}
$$

The stronger condition (29) in the B1 transition can be expressed as:

$-X_{\mathrm{sec}}<h v\left(1-2 T / T_{\mathrm{R}}\right)$

This inequality may not hold for very low light intensities (for correspondingly low $T_{\mathrm{R}}$ ) and/or for a very high secondary force. The lowest light absorption rate we used of $\alpha_{01}=1 \mathrm{~s}^{-1}$, required a secondary force of $0.4 \mathrm{~V}$ for the inequality (31) to become equation, i.e. to break down. For the light absorption rate of $\alpha_{01}=100 \mathrm{~s}^{-1}$, used in most of our computer experiments, required secondary force is even higher $0.7 \mathrm{~V}$. This can never happen, since maximal proton-motive force is about $0.2 \mathrm{~V}$. Therefore, connected maximums in the productive pathway will always occur, if we take care to perform free-energy transduction in the normal operating regime far from the static head state.

\subsection{The five-state model for chlorophyll based photoconversion}

A simplified five-state model (Fig. 4) for an-oxygenic chlorophyll-based bacterial photosynthesis is derived from the much more complete model used in the Bart van Rotterdam PhD thesis (1998). Selected states are chlorophyll ground state $\mathrm{P}$, chlorophyll excited state $\mathrm{P}^{*}$, and charge separated states $\mathrm{B} \equiv \mathrm{P}^{+} \mathrm{B}^{-}{ }_{\mathrm{A}}, \mathrm{H} \equiv \mathrm{P}^{+} \mathrm{H}^{-} \mathrm{A}$ and $\mathrm{Q} \equiv \mathrm{P}^{+} \mathrm{Q}^{-} \mathrm{A}$. The same kinetic model has been used for the simulation of the reaction center at steady-state by Lavergne and Joliot (1996).

As a Hill's diagram this model has seven transitions: L, D, B1,B2,B3,B4, S, and six cycles: a (L, D), b (L, B1, B2, S), c (L, B1, B2, B3, B4), d (D, B1, B2, S), e (D, B1, B2, B3, B4), and $\mathrm{f}(\mathrm{S}, \mathrm{B} 3, \mathrm{~B} 4)$. As in the three-state model there are only two operational fluxes and forces. The photon free energy (1) is the input force in cycles a and b, while cycles e and $\mathrm{f}$ are responsible for the creation of proton electrochemical gradient. In terms of transition affinities and currents (19):

$$
\begin{aligned}
T P= & A_{\mathrm{PP} *}(\mathrm{~L}) J(\mathrm{~L})+A_{\mathrm{P} * \mathrm{P}}(\mathrm{D}) J(\mathrm{D})+A_{\mathrm{P} * \mathrm{~B}}(\mathrm{~B} 1) J(\mathrm{~B} 1) \\
& +A_{\mathrm{BH}}(\mathrm{B} 2) J(\mathrm{~B} 2)+A_{\mathrm{HQ}}(\mathrm{B} 3) J(\mathrm{~B} 3) \\
& +A_{\mathrm{QP}}(\mathrm{B} 4) J(\mathrm{~B} 4)+A_{\mathrm{HP}}(\mathrm{S}) J(\mathrm{~S})
\end{aligned}
$$


Table 1

Optimized kinetic models of chlorophyll-based bacterial photosynthesis

\begin{tabular}{|c|c|c|c|c|c|c|c|}
\hline MODELS & 3-state I & 3-state II & 3-state III & 5-state I & 5-state II & 5-state III & 5-state IV \\
\hline O U T P U T & & & & & & & \\
\hline$\eta_{\text {opt }}(\%)$ & 17.97 & 17.52 & 16.68 & 13.57 & 17.46 & 18.83 & 19.04 \\
\hline$\eta_{\max }(\%)$ & 19.24 & 17.69 & 17.69 & 14.37 & 18.19 & 19.89 & 20.13 \\
\hline$\phi(\%)$ & 93.42 & 99.05 & 94.29 & 72.64 & 94.60 & 93.85 & 93.76 \\
\hline $\mathrm{A} / \mathrm{A}_{\text {oc }}(\%)$ & 84.41 & 92.23 & 85.32 & 91.96 & 87.84 & 87.07 & 87.10 \\
\hline $\mathrm{X}_{\mathrm{L}}(\mathrm{kJ} / \mathrm{mol})$ & 14.24 & 7.71 & 14.58 & 7.98 & 12.08 & 11.81 & 11.78 \\
\hline $\mathrm{J}(\mathrm{L})\left(\mathrm{s}^{-1}\right)$ & 2.72 & 65.16 & 68.04 & 81.83 & 71.92 & 2.88 & 3.38 \\
\hline $\mathrm{P}_{\text {tot }}\left(\mathrm{kJmol}^{-1} \mathrm{~T}^{-1} \mathrm{~s}^{-1}\right)$ & 0.68 & 17.90 & 18.88 & 23.56 & 19.78 & 0.73 & 0.84 \\
\hline $\mathrm{P}(\mathrm{L}) / \mathrm{P}\left(\mathrm{tot}^{\prime}\right)(\%)$ & 19.01 & 9.42 & 17.62 & 9.30 & 14.76 & 15.93 & 15.93 \\
\hline $\mathrm{k}_{1}(\mathrm{optimal})\left(\mathrm{s}^{-1}\right)$ & $1.42 \times 0^{11}$ & $1.04 \times 10^{10}$ & $1.65 \times 10^{11}$ & $2 \times 10^{10}$ & $4 \times 10^{11}$ & $4 \times 10^{11}$ & $4 \times 10^{11}$ \\
\hline $\mathrm{k}_{2}(\mathrm{optimal})\left(\mathrm{s}^{-1}\right)$ & 8.36 & 212 & 211 & $10^{11}$ & $4 \times 10^{11}$ & $4 \times 10^{11}$ & $4 \times 10^{11}$ \\
\hline $\mathrm{k}_{3}(\mathrm{optimal})\left(\mathrm{s}^{-1}\right)$ & - & - & - & $4.98 \times 10^{8}$ & $2.15 \times 10^{9}$ & $1.86 \times 10^{9}$ & $1.83 \times 10^{9}$ \\
\hline $\mathrm{k}_{4}(\mathrm{optimal})\left(\mathrm{s}^{-1}\right)$ & - & - & - & 403 & 254 & 10.08 & 21.50 \\
\hline $\mathrm{X}_{\mathrm{sec}}(\mathrm{mV})$ & -182 & -182 & -182 & -192 & -190 & -190 & -192 \\
\hline $\mathrm{I} \mathrm{N} \mathrm{P} \mathrm{U} \mathrm{T}$ & & & & & & & 4 \\
\hline$\alpha_{01}\left(\mathrm{~s}^{-1}\right)$ & 4 & 100 & 100 & 100 & 100 & 4 & 4 \\
\hline $\mathrm{K}(\mathrm{B} 1)$ & $2 \times 10^{19}$ & $2 \times 10^{19}$ & $2 \times 10^{19}$ & 0.21 & 4.8 & 4.8 & 4.8 \\
\hline $\mathrm{K}(\mathrm{B} 2)$ & 50 & 50 & 50 & $4.8 \times 10^{8}$ & 7.1 & 7.1 & 7.1 \\
\hline $\mathrm{K}(\mathrm{B} 4)$ & - & - & - & 5000 & 100 & 100 & 5000 \\
\hline $\mathrm{u}$ & 11 & 11 & 11 & 16 & 12 & 12 & 16 \\
\hline $\mathrm{k}_{\mathrm{s}}\left(\mathrm{s}^{-1}\right)$ & - & - & - & $10^{8}$ & $8 \times 10^{7}$ & $8 \times 10^{7}$ & $8 \times 10^{7}$ \\
\hline $\mathrm{k}_{\mathrm{d}}\left(\mathrm{s}^{-1}\right)$ & $10^{10}$ & $10^{8}$ & $10^{10}$ & $1.5 \times 10^{9}$ & $10^{9}$ & $10^{9}$ & $10^{9}$ \\
\hline
\end{tabular}

The application of Kirchhoff's junction and loop rule leads to relationships among currents and among affinities and forces as shown for the three-state model. Only the last recovery transition B4 is assumed to be the electrogenic step in which electron cycling is coupled to proton pumping. The $K_{4}$ equilibrium constant is then the function of electron

\section{Chlorophyll-Based 5-State Kinetic Model}

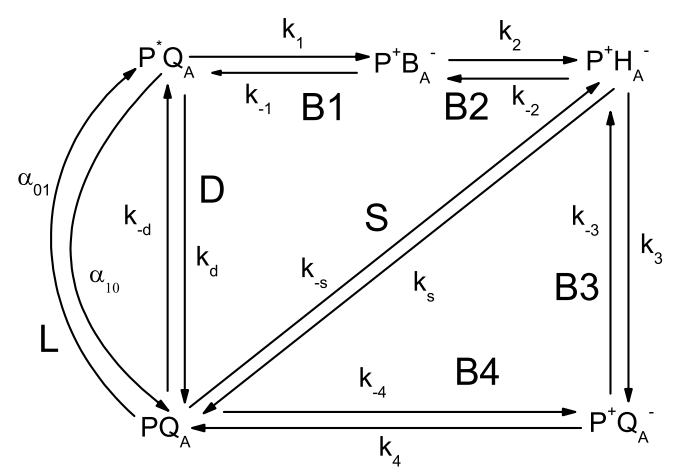

Fig. 4. The five-state kinetic model with a slip for the chlorophyll-based bacterial photosynthesis. Transitions leading to charge separation are B1, $\mathrm{B} 2, \mathrm{~B} 3$ and $\mathrm{B} 4$, while the slip transition $\mathrm{S}$ is non-productive transition. The electron transport is assumed coupled to proton pumping in the recovery $\mathrm{B} 4$ transition. The $\mathrm{Q}_{\mathrm{A}}$ is ubiquinone electron acceptor, the $\mathrm{B}_{\mathrm{A}}$ is accessory bacteriochlorophyll, while the $\mathrm{H}_{\mathrm{A}}$ is pheophytin. donor and acceptor concentrations and of the proton-motive force:

$K_{4}=k_{4} / k_{-4}=\exp \left(u+X_{\mathrm{sec}} / k_{\mathrm{B}} T\right)$

The $K(\mathrm{D})$ and $K(\mathrm{~L})$ constants are defined as for the three-state kinetic model too, and relationships among affinities and forces are used to find the equilibrium constants $K(\mathrm{~B} 3)$ and $K(\mathrm{~S})$. Using the diagram technique (Hill, 1977) it is now easy to derive the explicit dependence of state probabilities, transition flows, affinities, operational flows, overall efficiency and entropy production in each transition step as functions of rate constants.

Let us now use the data provided by Van Rotterdam (1998). Both initial transitions B1 and B1 transitions are regarded as close to equilibrium, with equilibrium constants $k_{1}=4.8$ and $k_{2}=7.1$, respectively. Entropy production associated with these transitions should be closer to the minimum value (zero) than to maximum. Initial relaxation from the excited state (exciton travelling, electron tunneling) is indeed nondissipative (Sumi, 2001) or low-dissipative event. It would make sense then to omit optimization for maximum entropy production in the transitions B1 and B2, and to perform optimization only in the recovery transitions B3 and B4. We performed such optimization by letting free only the rate constants in transitions B3 and B4, while all other rate and equilibrium constants were taken as pub- 


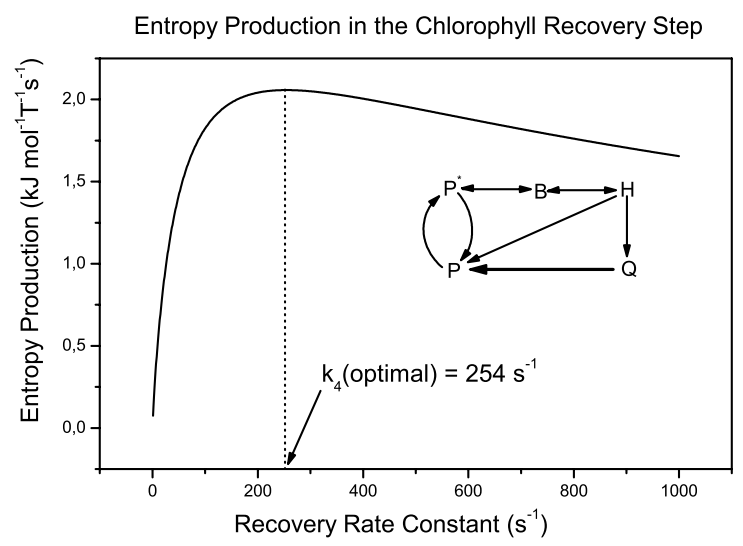

Fig. 5. The five-state kinetic model for chlorophyll-based photosynthesis has been optimized in the self-consistent manner so that entropy production is maximal in all non-slip irreversible dark transitions (B3 and B4 in the Fig. 4). Optimal overall efficiency is then $17.5 \%$, optimal affinity transfer efficiency is $87.8 \%$, and optimal photochemical yield is $94.6 \%$. The input constants, specified in the Table 1 (five-state II), are the same as previously used in this paper in simpler kinetic models and by Van Rotterdam (1998). Insert: the bold line in the kinetic model specifies recovery irreversible transition for which the entropy production dependence on the forward rate constant is shown in the main figure.

lished values (Van Rotterdam, 1998). Entropy production in the recovery transition (B4) is depicted in the Fig. 5, as the function of recovery rate constant $k_{4}$.

\subsection{Optimized chlorophyll-based photosynthetic models}

We explored power chanelling in different steady-states having in common maximum entropy production in all irreversible non-slip transitions. The results are sumarized in the Table 1.

Lavergne and Joliot (1996) parameters are used as the first five-state model. We took into account that first transition (B1) is essentially nondissipative with an equilibrium constant $k_{1}=0.21$, when rate constants provided in their paper are used. Since they do not provide the equilibrium constant for the B2 transition (it is actually varied in their work), we performed the optimization by varying the forward rate constants in B2, B3 and B4 transitions. The last three five-state models are all with Van Rotterdam parameters (1998), and only forward rate contants in B3 and B4 transitions are optimized. The photochemical yield is calculated as $J(\mathrm{~B} 2) / J(\mathrm{~L})$ in three-state models (Fig. 3) and as $J(\mathrm{~B} 4) / J(\mathrm{~L})$ in five-state models (Fig. 4).

A negative sign for $X_{\mathrm{sec}}$ means that photon-activated proton translocation (associated with the $J(\mathrm{~B} 2)$ or $J(\mathrm{~B} 4)$ current in our three-state and five-state kinetic scheme respectively) and transmembrane electrochemical potential are opposed to each other. Photon free-energy is being used to transport protons from bacterial interior to outside in the direction opposite to bacterial electric field, and this active transport process leads to additional charge separation and stronger electric field.
Optimal steady-state occupation of the excited state is in all models about 10 orders of magnitude less than the occupation of the ground state. The sum of maximal entropy productions in productive B transitions is in all models between 80 and $90 \%$ of the total entropy production.

Effective radiation temperature $T_{\mathrm{R}}$ is changed when light absorption rate is changed in the model. For instance, for the chlorophyll-based model the choice of $\alpha_{01}=1,4,100$ or $1000 \mathrm{~s}^{-1}$ leads to, respectively, $T_{\mathrm{R}}=827,888,1074$ and $1262 \mathrm{~K}$. We were interested if optimization for maximum entropy production in the charge separation pathway, would change the performance of the photoconverter, when light absorption rate is increased. Optimal values for the overall efficiency (21), thermodynamic force for light transitions (3) and affinity transfer efficiency $A / A_{\mathrm{oc}}$, are approximately constant over wide range of light intensities (Table 1). The $\mathrm{Q}$-state $\left(\mathrm{P}^{+} \mathrm{Q}^{-} \mathrm{A}\right)$ occupation also does not change when light intensity is varied (not shown), while the photochemical yield exhibits small decrease only for the lowest light intensities.

On the other hand, optimal values of the total entropy production and recovery rate constant $k_{4}$, are almost perfectly proportional to the photon absorption rate (compare five-state models II and III in the Table 1), while internal resistance analogue $X_{\mathrm{L}} / J_{\mathrm{L}}$ decreases 289 times when photon absorption increases 300 times (from $\alpha_{01}=1$ to $300 \mathrm{~s}^{-1}$ ). The excited state population and proton current $J(\mathrm{~B} 4)$ are also proportional to the photon absorption rate in optimized models (not shown). Once again we find that more complex nonlinear kinetic model maintains the same simple behaviour as the linearized two-state model (see Eqs. (10) and (11)).

It was also of interest to see if the optimized model can reproduce the backpressure regulation of energy transduction, namely that proton pumping is slowed down with an increase in the transmembrane potential (Van Rotterdam et al., 2001). Proton current indeed decreases, while the increase in the Q-state occupation parallels the increase in overall efficiency (21) at higher proton-motive force (Fig. 6). With increased absolute value for the secondary force from 92 to $208 \mathrm{mV}$ there was a small reduction in the photochemical yield from 95.4 to $94.3 \%$, and in the thermodynamic force $X_{\mathrm{L}}$ from 12.43 to $11.97 \mathrm{~kJ} \mathrm{~mol}^{-1}$, while the affinity transfer efficiency remained almost the same (it increased from 87.5 to $87.9 \%$ ).

\subsection{The five-state model for bacteriorhodopsin photosynthesis}

Since bacteriorhodopsin is the simplest photosynthetic system it is probably the best system where free-energy transduction theory and experiments may meet in the future. However, it is still far from clear which model for the bR-photocycle is the best (Hendler et al., 2001). The five-state kinetic model for bacteriorhodopsin photosynthesis (Fig. 7) was used in our 1987 paper dealing with 


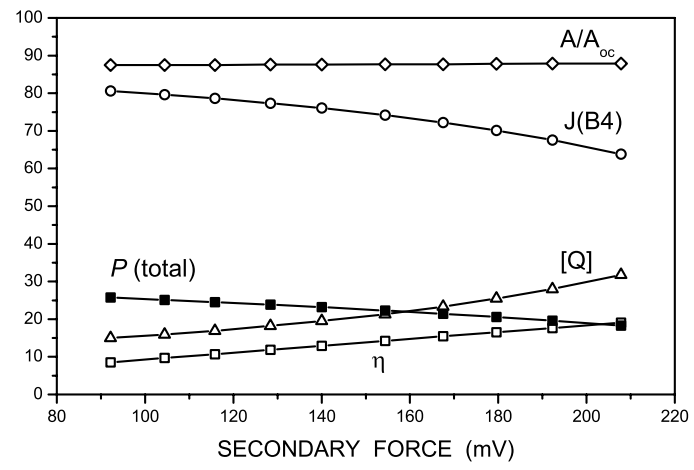

Fig. 6. The backpressure regulation of proton pumping by the protonmotive force. The optimization for maximal entropy production in irreversible transitions from productive pathway of the chlorophyll-based photosynthetic model leads to proton pump $\mathrm{J}(\mathrm{B} 4)$ slow-down (in $\mathrm{s}^{-1}$ ) and total entropy production (in $\mathrm{kJ} \mathrm{mol}^{-1} \mathrm{~T}^{-1} \mathrm{~s}^{-1}$ ) decrease, when absolute value of secondary force is increased. The occupation of the $\mathrm{Q}$ state $\left(\mathrm{P}^{+} \mathrm{Q}_{\mathrm{A}}{ }^{-}\right)$increases together with the optimal overall efficiency $\eta$, while optimal affinity transfer efficiency $A / A_{\mathrm{oc}}$ is nearly constant (these three values are in percentages).

dissipation and efficiency in nonlinear free-energy transduction (Juretic and Westerhoff, 1987). As in the case of the five-state model for chlorophyll-based photosynthesis (Fig. 6), there are seven possible transitions L, D, B1, B2, B3, B4 and S, and six cycles: a (L, D), b (L, B1, S), c (L, B1, B2, B3, B4), d (D, B1, S), e (D, B1, B2, B3, B4), and f (S, B2, B3, B4).

Since retinal chromofore absorbs light in the bacteriorhodopsin environment at $570 \mathrm{~nm}$, which is a lower wavelength than the absorbance maximum wavelength for chlorophyll, the input force is higher, so that maximal overall efficiency cannot be higher than $11.63 \%$. Stimulated emission rate constant $k_{\text {emm }}$ (also known as the Einstein coefficient A) was assumed to be essentially equall to $\alpha_{10}$. With its value chosen as $k_{\mathrm{emm}}=10^{9} \mathrm{~s}^{-1}$, the other Einstein's coefficient B was found from the requirement (Meszena and Westerhoff, 1999): $A / B=8 \pi v^{2} / c^{3}$.

Bacteriorhodopsin 5-State Kinetic Model

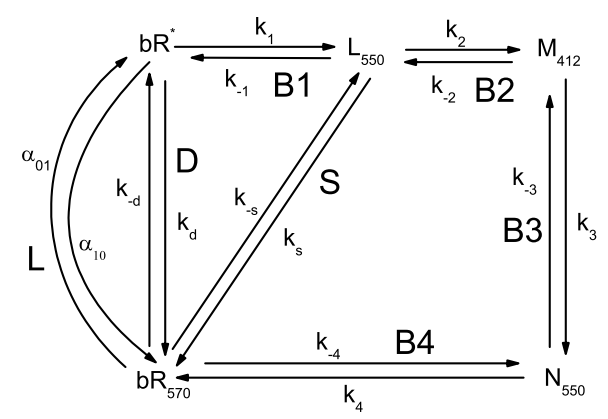

Fig. 7. The five-state kinetic model with a slip for the bacteriorhodopsin photocycle. The five states are the ground state $\left(b_{570}\right)$, excited state (bR*), and spectroscopic states $\mathrm{L}_{550}, \mathrm{M}_{412}$ and $\mathrm{N}_{550}$. In this model proton transfer is assumed to take place through B2 transition (proton release to extracytoplasmic space) and through B4 transition (proton absorption from the cytomplasmic space), Other symbols have the same meaning as in the Fig. 4.
The application of Kirchhoff's junction and loop rules gives relationships among fluxes and among affinities as in the three state model. The latter leads to relationship between equilibrium constants: $K(\mathrm{D})=K(\mathrm{~B} 1) K(\mathrm{~S})$. Equilibrium dissociation constants for the deprotonation and protonation reactions are assumed to be respectively:

$$
\begin{aligned}
& K_{\mathrm{E}}=K(\mathrm{~B} 2)\left[\mathrm{H}_{\mathrm{out}}^{+}\right]=K_{\mathrm{E}}^{0} \exp (F \Delta \Psi / 2 R T) \\
& K_{\mathrm{I}}=\left[\mathrm{H}_{\mathrm{in}}^{+}\right] / K(\mathrm{~B} 4)=K_{\mathrm{I}}^{0} \exp (-F \Delta \Psi / 2 R T)
\end{aligned}
$$

where $F$ is the Faraday constant, while $\Delta \Psi$ is the transmembrane potential.

This gives the correct expression for the secondary force as the proton-motive force:

$$
X_{\mathrm{sec}}=\Delta \mu_{\mathrm{H}^{+}}=F \Delta \Psi+R T \ln \left(\left[\mathrm{H}_{\mathrm{in}}^{+}\right] /\left[\mathrm{H}_{\mathrm{out}}^{+}\right]\right)
$$

if

$K(\mathrm{~B} 3) / K(\mathrm{~S})=K_{\mathrm{I}}^{0} / K_{\mathrm{E}}^{0}$

With a choice of essentialy irreversible reactions with high equilibrium constants, many different optimized kinetic models can be created (Table 2). The optimization procedure consisted in searching for models that have at the same time the free-energy transduction efficiency higher than $10 \%$ and maximal entropy production in each of four productive transitions B1, B2, B3 and B4 (Fig. 7). Since photon energy and external temperature $(298.16 \mathrm{~K})$ are kept constant, the ratio $h v / k_{\mathrm{B}} T=84.67$, is the same in all models. Secondary force is also kept at the constant value of $X_{\mathrm{sec}}=-18.84 \mathrm{~kJ} \mathrm{~mol}^{-1}(-195 \mathrm{mV})$.

The ground state is the most probable in all models ( 77 to $88 \%$ ), with the spectroscopic intermediates $\mathrm{M}_{412}$ and $\mathrm{N}_{550}$ (Fig. 7) following (up to 15\%), while the excited state [bR*] is $10^{8}$ to $10^{9}$ times less probable. However, higher excited state population can be best correlated with higher photochemical and overall efficiency. Higher overall efficiencies can also be correlated with lower thermodynamic force $X_{\mathrm{L}}$ and with lower percentage of dissipation in the $\mathrm{L}$ transition (Table 2).

It might be of interest to compare optimal forward kinetic constants (Table 2) with similar kinetic model $\mathrm{bR}^{*} \rightarrow \mathrm{L} \rightarrow \mathrm{M} \rightarrow \mathrm{O} \rightarrow \mathrm{bR}$, that also has four productive transitions and four time constants in the fast decay route of the bR-photocycle (Hendler et al., 2001). From Hendler et all modelling of experimental data, four relevant kinetic constants are:

$$
\begin{aligned}
& \left(k_{1}\right)_{\exp }=1.67 \times 10^{5} \mathrm{~s}^{-1} \quad\left(k_{2}\right)_{\exp }=3.03 \times 10^{4} \mathrm{~s}^{-1} \\
& \left(k_{3}\right)_{\exp }=556 \mathrm{~s}^{-1} \quad\left(k_{4}\right)_{\exp }=233 \mathrm{~s}^{-1}
\end{aligned}
$$

Except for the much shorter time constant for the B1 transition, optimal rate constants from optimized kinetic models I to VII (Table 2.) span the range where experimental values (37) are found also. As for the kinetic models for chlorophyll-based bacterial photosynthesis we noticed 
Table 2

Optimized kinetic models of the bacteriorhodopsin photocycle

\begin{tabular}{|c|c|c|c|c|c|c|c|}
\hline MODELS & $\mathrm{I}$ & II & III & IV & $\mathrm{V}$ & VI & VII \\
\hline \multicolumn{8}{|l|}{ O U T P U T } \\
\hline$\eta_{\text {opt }}(\%)$ & 10.27 & 10.35 & 10.17 & 10.07 & 10.08 & 10.34 & 10.52 \\
\hline$\eta_{\max }(\%)$ & 10.40 & 11.11 & 10.28 & 10.32 & 10.13 & 10.46 & 10.74 \\
\hline$\phi(\%)$ & 90.44 & 91.21 & 89.55 & 88.73 & 90.96 & 93.28 & 94.90 \\
\hline $\mathrm{A} / \mathrm{A}_{\mathrm{oc}}(\%)$ & 97.44 & 98.86 & 96.69 & 94.89 & 95.59 & 97.37 & 97.82 \\
\hline $\mathrm{X}_{\mathrm{L}}(\mathrm{kJ} / \mathrm{mol})$ & 4.26 & 1.89 & 5.50 & 8.49 & 7.32 & 4.36 & 3.61 \\
\hline $\mathrm{J}(\mathrm{L})\left(\mathrm{s}^{-1}\right)$ & 14.59 & 9.50 & 15.61 & 14.94 & 73.21 & 71.75 & 60.07 \\
\hline $\mathrm{P}_{\text {tot }}\left(\mathrm{kJmol}^{-1} \mathrm{~T}^{-1} \mathrm{~s}^{-1}\right)$ & 7.28 & 4.74 & 7.80 & 7.48 & 37.52 & 36.66 & 30.63 \\
\hline $\mathrm{P}(\mathrm{L}) / \mathrm{P}($ tot $)(\%)$ & 2.85 & 1.27 & 3.69 & 5.68 & 4.79 & 2.86 & 2.38 \\
\hline $\mathrm{k}_{1}($ optimal $)\left(\mathrm{s}^{-1}\right)$ & $4.49 \times 10^{9}$ & $1.18 \times 10^{9}$ & $8.1 \times 10^{9}$ & $2.87 \times 10^{10}$ & $1.81 \times 10^{10}$ & $4.73 \times 10^{9}$ & $3.22 \times 10^{9}$ \\
\hline $\mathrm{k}_{2}($ optimal $)\left(\mathrm{s}^{-1}\right)$ & $1.23 \times 10^{4}$ & $9.3 \times 10^{4}$ & 970 & $1.12 \times 10^{6}$ & $1.07 \times 10^{7}$ & $2.01 \times 10^{4}$ & 4590 \\
\hline $\mathrm{k}_{3}$ (optimal) $\left(\mathrm{s}^{-1}\right)$ & 284 & 186 & 301 & 85.6 & 430 & 1000 & 812 \\
\hline $\mathrm{k}_{4}($ optimal $)\left(\mathrm{s}^{-1}\right)$ & 208 & 137 & 221 & 183 & 919 & 1060 & 425 \\
\hline \multicolumn{8}{|l|}{ I N P U T } \\
\hline$\alpha_{01}\left(s^{-1}\right)$ & 20 & 20 & 20 & 20 & 100 & 100 & 100 \\
\hline $\mathrm{K}(\mathrm{B} 1)$ & $10^{8}$ & $10^{5}$ & $10^{11}$ & $10^{13}$ & $10^{13}$ & $10^{8}$ & $10^{8}$ \\
\hline K(B2) & $10^{8}$ & $10^{11}$ & $10^{5}$ & $10^{11}$ & $10^{11}$ & $10^{11}$ & $10^{15}$ \\
\hline $\mathrm{K}(\mathrm{B} 4)$ & $10^{8}$ & $10^{8}$ & $10^{8}$ & $10^{7}$ & $10^{7}$ & $10^{8}$ & $10^{4}$ \\
\hline $\mathrm{K}_{\mathrm{I}}^{0} / \mathrm{K}_{\mathrm{E}}^{0}$ & $5 \times 10^{-20}$ & $5 \times 10^{-23}$ & $5 \times 10^{-17}$ & $5 \times 10^{-22}$ & $5 \times 10^{-22}$ & $5 \times 10^{-23}$ & $5 \times 10^{-23}$ \\
\hline $\mathrm{k}_{\mathrm{s}}\left(\mathrm{s}^{-1}\right)$ & 1000 & 100 & 100 & $10^{5}$ & $10^{6}$ & 1000 & 100 \\
\hline$k_{d}\left(s^{-1}\right)$ & $10^{8}$ & $10^{8}$ & $10^{8}$ & $10^{9}$ & $10^{8}$ & $10^{8}$ & $10^{8}$ \\
\hline
\end{tabular}

that greater slip my be beneficial in greatly increasing optimal forward rate constants next to slip transition (Tables 1 and 2).

The backpressure regulation (Van Rotterdam et al., 2001) was present in all kinetic models from the Table 2. As an example, as we increased the secondary force from 95 to $195 \mathrm{mV}$ in the model VII, optimal values of recovery constant $k_{4}$, recovery current $J(\mathrm{~B} 4)$ and of total entropy production, decreased $37 \%, 6 \%$ and $11 \%$ respectively. Optimal photochemical yield decreased slightly ( $2 \%$ or less), while optimal affinity transfer efficiency remained the same.

\section{Discussion}

In this work we examined the entropy production at a very detailed level, namely in each transition between neighboring states that form the photosynthetic cycle. Energy and flux balance analysis (Beard et al., 2002) are applied in the steady-state just as it is done in electrical circuits (Kirchhoff's laws). In linear electrical circuits steady-state currents are distributed to achieve maximum entropy production (see Introduction). Taking into account that a cell, interacting with its environment, can vary the process parameters, we extended the maximum entropy production principle (Jeans, 1923; Onsager, 1931a,b; Kohler, 1948; Ziman, 1956), proposing that evolution developed mechanisms for adjusting rate constants in irreversible transitions of the productive pathway toward their optimal values, making entropy pro- duction maximal in these transitions. Only the transitions leading to creation of the electrochemical proton gradient are potentially productive. Efficient power transfer to these transitions is the consequence of proposed maximum entropy production principle in all of considered kinetic models for chlorophyll-based and bacteriorhodopsin-based photosynthesis. The mechanism of how rate constants can change is left open. Random mutations can certainly change the microenvironment of integral membrane protein where charge separation takes place, so that rate constant change can be connected to biological evolution and selection against changes that decrease power transfer into the productive pathway. The short term self-regulation of the photoexcited electron/proton flux is also possible through light-induced structural rearrangements of the macromolecule.

Since photosynthesis is essential for almost all life on earth, understanding its thermodynamics will certainly help in understanding the physical background for biological evolution. In physics, an entropy production value for a system is a convenient measure for the speed of thermodynamic evolution of that system, i.e. how far is that system from the thermodynamic equilibrium (when entropy production vanishes). Our proposal that entropy production is maximal in all initial irreversible charge-separating steps produces realistic performance in simple photosynthetic models, which brings biological evolution in synergy with thermodynamic evolution: the former accelerates the latter in a coupled self-amplifying process. 
Several important predictions are obtained by using our proposal. The optimal values of thermodynamic parameters, such as the overall efficiency, photochemical yield, and affinity transfer efficiency, are nearly constant for a wide range of light intensities. It follows that a photoconverter operating in the optimal regime would be insensitive to the energy input into the system. Optimal proton current and free-energy dissipation increase with higher light absorption rate and decrease with stronger proton-motive force. Optimal coupling between electron and proton current, as expressed through the recovery rate constant $k_{4}$, also increases in parallel with higher light absorption rate, and decreases with stronger secondary force. Optimal photochemical yield decreases slightly with stronger secondary force. Some evidence for the reduction in photochemical yield due to stronger membrane potential, opposing light-induced proton active transport, has been indeed observed in experiments (Gopher et al., 1985; Lao et al. 1993). The back-pressure effect of proton pump acceleration in the presence of weaker secondary force (Fig. 6) has also been reported (Van Rotterdam et al., 2001). The back-pressure control of photosynthetic free-energy transduction and maximum thermodynamic (overall) efficiency limited to less than $20 \%$ is a common feature of reconstituted liposomal systems (Van Rotterdam et al., 2001) and our optimized models. Additional feature of optimized models common with observed kinetics of photochemical cycles is a spread of eight orders of magnitude, or even more, between the fastest (relaxation from the excited state) and the slowest (recovery to ground state) rate constant.

Another important prediction is that maximizing entropy production, in the case of nonlinear current-force relationship, has a definite evolutionary advantage over linear mode of operation, because most of the power (more than 90\%) is than channelled in the charge-separation pathway. This advantage of a nonlinear mode is used both in sollar cells and in photosynthesis. It is likely to be common to all biological free-energy converters. This conclusion is the opposite of the one reached by Stucki et al. (1983), namely, that the linear mode of operation of biological energy converters is superior by a very large factor.

In the linear range the claim that photosynthesis operates in the minimal entropy production mode (Andriesse and Hollestelle, 2001) is flawed, because it is based on calculations (Andriesse, 2000), that can lead only to thermodynamic equilibrium, i.e. to zero dissipation state (Juretic, 2002). The zero dissipation state of the whole system (when all forces are unconstrained and allowed to vanish) represents the thermodynamic death of a system, or thermodynamic equilibrium when free-energy transduction is impossible. Knowing that free-energy transduction in chloroplast or photosynthetic bacteria is at last $10^{5}$ times more intensive than in the Sun (Metzner, 1984), makes it hard to believe that zero dissipation state can be highly relevant for photosynthesis, as recently implicitly claimed (Andriesse and Hollestelle, 2001). In the linear mode entropy production is minimal, but different from zero, in the static head state. However, the static head state itself is the zero efficiency state with respect to free-energy transduction. In the nonlinear mode entropy production is also extremely small near static head state but not minimal (not shown). In addition, such a state can not be reached in practice (when leaks are not present), because membrane potential required for vanishing net proton flux would be so large to cause the dielectric breakdown of a membrane (to be published).

In conclusion, we have shown that kinetic models for efficient steady-state photosynthesis can easily be built if Kirchoff's loop and junction rules are used and maximum entropy production is required in irreversible dark transitions (excluding slip). The sum of maximal entropy productions in irreversible charge transfer steps is the major contribution to the total entropy production in all our optimized kinetic models and the performance of models is in general agreement with experimental observations. Optimized nonlinear models have high free-energy/affinity transmision efficiency, often exceeding $90 \%$, which is a definite evolutionary advantage over maximum of $50 \%$ allowed by the maximum power transfer theorem in a linear mode.

\section{Acknowledgements}

Thanks are due to Hans Westerhoff, Bart van Rotterdam and Richard Hendler for allowing us to read their recent publications and to Croatian Ministry of Science and Technology for financial support given to the D.J. with grant no. 0177163.

\section{References}

Adami, C., Ofria, C., Collier, T.C., 2000. Evolution of biological complexity. Proc. Natl. Acad. Sci. USA 97, 4463-4468.

Andresen, B., Shiner, J.S., Uehlinger, D.E., 2002. Allometric scaling and maximum efficiency in physiological eigen time. Proc. Natl. Acad. Sci. USA 99, 5822-5824.

Andriesse, C.D., 2000. On the relation between stellar mass loss and luminosity. Astronom. J. 539, 364-365.

Andriesse, C.D., Hollestelle, M.J., 2001. Minimum entropy production in photosynthesis. Biophys. Chem. 90, 249-253.

Beard, D.A., Liang, S.D., Qian, H., 2002. Energy balance for analysis of complex metabolic networks. Biophys. J. 83, 79-86.

Boylestad, R., 1999. Introductory Circuit Analysis, Prentice-Hall, Upper Saddle River, NJ.

Cho, H.M., Mancino, L.J., Blankenship, R.E., 1984. Light saturation curves and quantum yields in reaction centers from photosynthethic bacteria. Biophys. J. 45, 455-461.

De Donder, T., Van Rysselberghe, P., 1936. Thermodynamic Theory of Affinity, Stanford University Press, Stanford.

Dewar, R., 2003. Information theory explanation of the fluctation theorem, maximum entropy production and self-organized criticality in non-equilibrium stationary states. J. Phys. A Math. Gen. 36, 631-641.

Green, M.A., 1982. Solar Cells. Operating Principles, Technology, and System Applications. Pretince-Hall, Englewood Cliffs, NJ. 
Gopher, A., Blatt, Y., Schonfeld, M., Okamura, M.Y., Feher, G., Montal, M., 1985. The effect of an applied electric field on the charge recombination kinetics in reaction centers reconstituted in planar lipid bilayers. Biophys. J. 48, 311-320.

Heinrich, R., Hoffman, E., 1991. Kinetic parameters of enzymatic reactions in states of maximal activity; An evolutionary approach. J. Theor. Biol. 151, 249-283.

Hendler, R.W., Shrager, R.I., Bose, S., 2001. Theory and practice for finding a correct kinetic model for the bacteriorhodopsin photocycle. J. Phys. Chem. B. 105, 3319-3328.

Hill, T.L., 1977. Free Energy Transduction in Biology. The Steady State Kinetic and Thermodynamic Formalism. Academic Press, New York.

Hill, T.L., 1989. Free Energy Transduction and Biochemical Cycle Kinetics. Springer-Verlag, New York.

Hunt, K.L.C., Hunt, P.M., 1987. Dissipation in steady-states of chemical systems and deviations from minimum entropy production. Physica 147A, 48-60.

Jeans, J.H., 1923. The Mathematical Theory of Electricity and Magnetism, 4th ed. Cambridge Univerity Press, Cambridge, pp. 322-323.

Juretić, D., 1983. The thermodynamic and kinetic limits on the process of free energy storage by photosynthetic systems. Croat. Chem. Acta $56,383-387$.

Juretic, D., 1984. Efficiency of free energy transfer and entropy production in photosynthetic systems. J. Theor. Biol. 106, 315-327.

Juretić, D., 1992. Membrane free-energy converters: the benefits of intrinsic uncoupling and non-linearity. Acta Pharm. 42, 373-376.

Juretic, D., 2002. Comment on 'Minimum entropy production in photosynthesis', BioComplexity 1. http://jjj.biochem.sun.ac.za/home/ HW/BIOCOMPLEXITYWEB/Jureticfinal.htm

Juretić, D., Westerhoff, H.V., 1987. Variation of efficiency with free-energy dissipation in models of biological energy transduction. Biophys. Chem. 28, 21-34.

Knox, R.S., 1977. Photosynthetic efficiency and exciton transfer and trapping. In: Barber, J. (Ed.), Primary Processes in Photosynthesis, vol. 2. Elsevier, New York, pp. 55-97.

Kohler, M., 1948. Behandlung von Nichtgleichgewichtsvorgängen mit Hilfe eines Extremalprinzips. Z. Phys. 124, 772-789.

Kubo, R., 1976. Thermodynamics. North Holland, Amsterdam.

Lanyi, J.K., Luecke, H., 2001. Bacteriorhodopsin. Curr. Opin. Struct. Biol. $11,415-419$.

Lao, K., Franzen, S., Stanley, R.J., Lambright, D.G., Boxer, S.G., 1993. Effects of applied electric field on the quantum yields of initial electron transfer steps in bacterial photosynthesis. 1. Quantum yield failure. J. Phys. Chem. 97, 13165-13171.

Lavergne, J., Joliot, P., 1996. Dissipation in bioenergetic electron transfer chains. Photosynth. Res. 48, 127-138.

Lavergne, J., Joliot, P., 2000. Thermodynamics of the excited states of photosynthesis. In: BTOL-Bioenergetics, Ch. 2 (http://www.biophysics. org/btol/bioenerg.html).
Meszena, G., Westerhoff, H.V., 1999. Non-equilibrium thermodynamics of light absorption. J. Phys. A.: Math. Gen. 32, 301-311.

Metzner, H., 1984. Bioelectrochemistry of photosynthesis: a theoretical approach. Bioelectrochem. Bioenerg. 13, 183-190.

Onsager, L., 1931a. Reciprocal relations in irreversible processes I. Phys. Rev. 37, 405-426.

Onsager, L., 1931b. Reciprocal relations in irreversible processes II. Phys. Rev. 38, 2265-2279.

Paltridge, G.W., 1979. Climate and thermodynamic systems of maximum dissipation. Nature 279, 630-631.

Pettersson, G., 1996. A new approach for determination of the selectively favored kinetic design of enzyme reactions. J. Theor. Biol. 183, 179183.

Prigogine, I., 1967. Thermodynamics of Irreversible Processses. Interscience, New York.

Rebhan, E., 1990. Maximum entropy production far from equilibrium: the example of strong shock waves. Phys. Rev. A 42, 781-788.

Sawada, Y., 1981. A thermodynamic variational principle in nonlinear non-equilibrium phenomena. Prog. Theor. Phys. 66, 68-76.

Schneider, T.D., 2000. Evolution of biological information. Nucleic Acids Res. 28, 2794-2799.

Stucki, J.W., 1980. The optimal efficiency and the economic degrees of coupling of oxidative phosphorylation. Eur. J. Biochem. 109, 269-283.

Stucki, J.W., Compiani, M., Caplan, S.R., 1983. Efficiency of energy conversion in model biological pumps. Optimization by linear nonequilibrium thermodynamic relations. Biophys. Chem. 18, 101-109.

Sumi, H., 2001. Bacterial photosynthesis begins with quantum-mechanical coherence. Chem. Rec. 1, 480-493.

Swenson, R., 1989. Emergent attactors and the law of maximum entropy production: Foundations to a theory of general evolution. Syst. Res. 6, 187-197.

Ulanowicz, R.E., Hannon, B.M., 1987. Life and the production of entropy. Proc. R. Soc. Lond. B 232, 181-192.

Van Rotterdam, B., 1998. Control of Light-Induced Electron Transfer in Bacterial Photosynthesis, PhD thesis, University of Amsterdam.

Van Rotterdam, B.J., Westerhoff, H.V., Visschers, R.W., Bloch, D.A., Hellingwerf, K.J., Jones, M.R., Crielaard, W., 2001. Pumping capacity of bacterial reaction centers and backpressure regulation of energy transduction. Eur. J. Biochem. 268, 958-970.

Van Rotterdam, B.J., Crielaard, W., van Stokkum, I.H.M., Hellingwerf, K.J., Westerhoff, H.V., 2002. Simplicity in complexity: the photosynthetic reaction center performs as a simple $0.2 \mathrm{~V}$ battery. FEBS Lett. 510, 105-107.

Ziegler, H., 1972. Systems with internal parameters obeying the orthogonality condition. J. Appl. Math. Phys. 23, 553-566.

Ziman, J.M., 1956. The general variational principle of transport theory. Can. J. Phys. 34, 1256-1273. 